\title{
Robust Fault Detection for Uncertain System with Communication Delay and Measurement Missing
}

\author{
Chunpeng Wang* \\ School of Electrical Engineering, Binzhou Polytechnic, Binzhou 256603, China \\ fuwa08@163.com
}

Keywords: robust fault detection filter, communication delays, missing measurements, $H_{\infty}$ filtering, linear matrix inequality.

\begin{abstract}
The robust fault detection problem is investigated for a class of norm-bounded uncertain systems with communication delays and multiple measurement packet dropouts. The networked time-delay and missing measurements are modeled as a linear function of the stochastic variable satisfying Bernoulli random binary distribution. A robust fault detection filter based on $H_{\infty}$ filtering is designed to make residual error system be exponentially mean-square stable and satisfy a prescribed disturbance attenuation level. A sufficient condition is derived in terms of linear matrix inequalities. Numerical examples are given to illustrate the effectiveness of the proposed method.
\end{abstract}

\section{Introduction}

The robust fault detection method based on $H_{\infty}$ filtering ${ }^{[1-7]}$ which is clear in concept and simple in implementation has attracted much attention of researchers and has gained rather rich research results. However, this method is built upon precise mathematical model. In practice, modeling errors and unknown inputs in complex engineering systems are unavoidable. As results, the research of robust fault detection for a class of uncertain systems is receiving extensive attention. The design of robust fault diagnosis filter for a class of singular time-delay systems subject to parameter uncertainties and disturbances has been studied in [3, 4]. The problem of the robust fault detection for a class of linear uncertain systems via LMI has been studied in [5]. The design of fault detection filter for a class of uncertain systems with one-step delay has been investigated in [6], however, the impact of missing measurements on the fault detection performance has been not considered in this paper.

At present, with the general application of network in industrial production, the phenomenon of time-delay and missing measurements has been widespread in the process of data transmission. In the case of incomplete measurement information, the problem of fault detection has become the focus of current research. In [7, 8], the problem of the filtering and fault detection with random time-delays and measurement packet dropouts has been discussed respectively. The design of fault detection filter for linear discrete-time systems with random time-delays and measurement packet dropouts has been studied in [9]. Parity space-based and observer-based, respectively, haven been used to solve a class of Markov jump systems with measurement packet dropouts in [10,11]. However, the impact of system modeling errors on fault detection is not considered in the above literatures. Therefore, the robust fault detection problem of linear uncertain systems with data loss is still to be studied.

In this paper, the robust fault detection problem is investigated for a class of norm-bounded uncertain systems with communication delays and multiple measurement packet dropouts. The networked time-delay and missing measurements are described by a linear function of the stochastic variable satisfying Bernoulli random binary distribution. By using $H_{\infty}$ control theory, the robust fault detection filter (RFDF) design can be formulated as an $H_{\infty}$ filtering problem. A sufficient condition for the existence of the desired fault detection filter is established in terms of linear matrix 
inequalities (LMIs), and a numerical example is provided to illustrate the effectiveness and applicability of the proposed design method.

\section{Problem formulation}

Consider the following class of uncertain discrete-time linear systems:

$$
\left\{\begin{array}{l}
x(k+1)=(A+\Delta A) x(k)+\left(B_{d}+\Delta B_{d}\right) d(k)+\left(B_{f}+\Delta B_{f}\right) f(k) \\
y(k)=C x(k)+D_{d} d(k)+D_{f} f(k)
\end{array}\right.
$$

where $x(k) \in R^{n}$ is the state vector; $y(k) \in R^{q}$ is the signal to be estimated; $d(k) \in R^{p}$ is the unknown disturbance input; $f(k) \in R^{r}$ is the fault to be detected; $A, B_{d}, B_{f}, C, D_{d}$ and $D_{f}$ are known real constant matrices with appropriate dimensions. $\Delta A, \Delta B_{d}$ and $\Delta B_{f}$ are unknown matrices describing parameter uncertainties which are assumed to be of the form:

$$
\Delta A=H F(k) G_{1}, \quad \Delta B_{d}=H F(k) G_{2}, \quad \Delta B_{f}=H F(k) G_{3}
$$

where $H, G_{1}, G_{2}$ and $G_{3}$ are known real constant matrices of appropriate dimensions, and $F(k)$ represents an unknown real-valued time-varying matrix satisfying $F^{T}(k) F(k) \leq I$.

The insertion of network cable between measurement node and fault detection filter causes random time-delay and missing phenomena. Furthermore, the measurement can be described by

$$
\psi(k)=\delta_{0}(k) y(k)+\delta_{1}(k) y(k-1)+\delta_{2}(k) \psi(k-1)
$$

where $\psi(k) \in R^{q}$ is the measured output, and the stochastic variable $\delta_{i}(k) \in R$ is a Bernoulli distributed white sequence with

$$
\left\{\begin{array}{l}
\operatorname{Prob}\{\delta(k)=i\}=E\left\{\delta_{i}(k)=1\right\}=\delta_{i} \\
\operatorname{Prob}\{\delta(k) \neq i\}=E\left\{\delta_{i}(k)=0\right\}=1-\delta_{i} \quad 0 \leq i \leq 2 \\
E\left\{\left(\delta_{i}(k)-\delta_{i}\right)\right\}=\delta_{i}\left(1-\delta_{i}\right)=\alpha_{i}
\end{array}\right.
$$

and $\delta_{i} \in R$ is a known positive scalar.

The core module of the fault detection based on $H_{\infty}$ filtering is the generation of residuals. In the case of system (1) is said to be asymptotically stable, consider the following RFDF for system with communication delays and multiple measurement packet dropouts:

$$
\left\{\begin{array}{l}
\hat{x}(k+1)=A_{F} \hat{x}(k)+B_{F} \psi(k) \\
r(k)=C_{F} \hat{x}(k)
\end{array}\right.
$$

where $\hat{x}(k) \in R^{n}$ is the state estimate, $r(k) \in R^{r}$ is the so-called residual that is compatible with the fault vector, and $A_{F} \in R^{n \times n}, B_{F} \in R^{n \times p}$ and $C_{F} \in R^{p \times n}$ are filter parameters to be determined.

Define the augmented state vector $\xi(k)=\left[\begin{array}{ll}x^{T}(k) & \hat{x}^{T}(k)\end{array}\right]^{T}$.

Denote $\omega(k)=\left[\begin{array}{ll}d^{T}(k) & f^{T}(k)\end{array}\right]^{T}, r_{e}(k)=r(k)-f(k)$, the augmented system formed by system (1), (3) and the filter (5) can be expressed by

where

$$
\left\{\begin{array}{l}
\xi(k+1)=A_{1} \xi(k)+A_{2} \xi(k-1)+B_{1} \omega(k)+B_{2} \omega(k-1) \\
r_{e}(k)=C_{1} \xi(k)+D_{1} \omega(k)
\end{array}\right.
$$

$A_{1}=A_{11}+\Delta A_{11}+\left(\delta_{0}(k)-\delta_{0}\right) A_{12}+\left(\delta_{2}(k)-\delta_{2}\right) A_{13}$,

$A_{11}=\left[\begin{array}{cc}A & 0 \\ \delta_{0} B_{F} C & A_{F}+\delta_{2} I\end{array}\right], A_{12}=\left[\begin{array}{cc}0 & 0 \\ B_{F} C & 0\end{array}\right], A_{13}=\left[\begin{array}{ll}0 & 0 \\ 0 & I\end{array}\right], \Delta A_{11}=\left[\begin{array}{cc}\Delta A & 0 \\ 0 & 0\end{array}\right]=\left[\begin{array}{c}H \\ 0\end{array}\right] F(k)\left[\begin{array}{ll}G_{1} & 0\end{array}\right]=\bar{H} F(k) \bar{G}_{1}$,

$A_{2}=A_{21}+\left(\delta_{1}(k)-\delta_{1}\right) A_{22}+\left(\delta_{2}(k)-\delta_{2}\right) A_{23}, A_{21}=\left[\begin{array}{cc}0 & 0 \\ \delta_{1} B_{F} C & -\delta_{2} A_{F}\end{array}\right], A_{22}=\left[\begin{array}{cc}0 & 0 \\ B_{F} C & 0\end{array}\right], A_{23}=\left[\begin{array}{cc}0 & 0 \\ 0 & -A_{F}\end{array}\right]$,

$B_{1}=B_{11}+\Delta B_{11}+\left(\delta_{0}(k)-\delta_{0}\right) B_{12}$ 


$$
\begin{aligned}
& B_{11}=\left[\begin{array}{cc}
B_{d} & B_{f} \\
\delta_{0} B_{F} D_{d} & \delta_{0} B_{F} D_{f}
\end{array}\right], B_{12}=\left[\begin{array}{cc}
0 & 0 \\
B_{F} D_{d} & B_{F} D_{f}
\end{array}\right], \Delta B_{11}=\left[\begin{array}{cc}
\Delta B_{d} & \Delta B_{f} \\
0 & 0
\end{array}\right]=\left[\begin{array}{c}
H \\
0
\end{array}\right] F(k)\left[\begin{array}{ll}
G_{2} & G_{3}
\end{array}\right]=\bar{H} F(k) \bar{G}_{2}, \\
& B_{2}=B_{21}+\left(\delta_{1}(k)-\delta_{1}\right) B_{22}, B_{21}=\left[\begin{array}{cc}
0 & 0 \\
\delta_{1} B_{F} D_{d} & \delta_{1} B_{F} D_{f}
\end{array}\right], B_{22}=\left[\begin{array}{cc}
0 & 0 \\
B_{F} D_{d} & B_{F} D_{f}
\end{array}\right], C_{1}=\left[\begin{array}{ll}
0 & C_{F}
\end{array}\right], D_{1}=\left[\begin{array}{ll}
0 & -I
\end{array}\right] .
\end{aligned}
$$

After the process, the fault detection problem stated above has been transformed into the robust filtering problem with networked time-delay and missing measurements. More specifically, the desigened filter (5) such that he following requirements are satisfied simultaneously: for a given prescribed scalar $\gamma>0$, the system (6) is exponentially mean-square stable and

$$
E\left\|r_{e}(k)\right\|_{2}<\gamma E\|\omega(k)\|_{2}
$$

The finial important task of fault detections is the residual evaluation. Based on the designed fault detection system, the residual evaluation function $J(k)$ and the threshold $J_{\text {th }}{ }^{[4]}$ are determined as follows:

$$
J(k)=\left\{\sum_{k=k_{0}}^{k_{0}+N} r^{T}(k) r(k)\right\}^{1 / 2}, \quad J_{t h}=\sup _{d(k) \in l_{2}, f(k)=0} E\{J(k)\}
$$

where $k_{0}$ denotes the initial evaluation time instant, $N$ denotes the length of time window.

Based on the above, the fault can be detected by using the following logical relationship:

$$
\left\{\begin{array}{l}
J(k)>J_{t h} \Rightarrow \text { abnormal } \Rightarrow \text { fault } \\
J(k) \leq J_{t h} \Rightarrow \text { normal }
\end{array}\right.
$$

\section{Design of robust fault detection filter}

Theorem 1: For a given scalar $\gamma>0$, the system (6) is exponentially mean-square stable and achieves the $H_{\infty}$-norm constraint (7) for all nonzero $\omega(k)$, if there exist positive definite matrices $P$ and $Q$ satisfying (10).

$$
\left[\begin{array}{cccccccccc}
\psi_{1} & * & * & * & * & * & * & * & * & * \\
0 & -Q & * & * & * & * & * & * & * & * \\
\varepsilon \bar{G}_{2}^{T} \bar{G}_{1} & 0 & \psi_{2} & * & * & * & * & * & * & * \\
0 & 0 & 0 & -\gamma^{2} I & * & * & * & * & * & * \\
P A_{11} & P A_{21} & P B_{11} & P B_{21} & -P & * & * & * & * & * \\
\alpha_{0} P A_{12} & 0 & \alpha_{0} P B_{12} & 0 & 0 & -\alpha_{0} P & * & * & * & * \\
0 & \alpha_{1} P A_{22} & 0 & \alpha_{1} P B_{22} & 0 & 0 & -\alpha_{1} P & * & * & * \\
\alpha_{2} P A_{13} & \alpha_{2} P A_{23} & 0 & 0 & 0 & 0 & 0 & -\alpha_{2} P & * & * \\
C_{1} & 0 & D_{1} & 0 & 0 & 0 & 0 & 0 & -I & * \\
0 & 0 & 0 & 0 & \bar{H}^{T} P & 0 & 0 & 0 & 0 & -\varepsilon I
\end{array}\right]<0
$$

where $\psi_{1}=-P+Q+\varepsilon \bar{G}_{1}^{T} \bar{G}_{1}, \psi_{2}=-\gamma^{2} I+\varepsilon \bar{G}_{2}^{T} \bar{G}_{2}$, and $\varepsilon \in R$ is any positive scalar.

Remark 1: The proof of Theorem 1 would require to be combined with definition in [12], lemma 2 in [1], lemma 5 in[2] and lemma 1 in [13]. The specific proof process would be omitted.

On the basis of Theeorem 1 , the detailed design method of the robusr fault detection filter would be derived.

Corollary 1: For a given scalar $\gamma>0$, the system (6) is exponentially mean-square stable and achieves the $H_{\infty}$-norm constraint (7) for all nonzero $\omega(k)$, if there exist positive definite matrices $W, Y, Z_{1}$ and $Z_{3}$, matrices $Z_{2}, M, N$ and $S$ satisfying (11). 
where

$$
\left[\begin{array}{ccccccccc}
\psi_{11} & * & * & * & * & * & * & * & * \\
0 & \psi_{22} & * & * & * & * & * & * & * \\
\psi_{31} & 0 & \psi_{33} & * & * & * & * & * & * \\
0 & 0 & 0 & -\gamma^{2} I & * & * & * & * & * \\
\psi_{51} & \psi_{52} & \psi_{53} & \psi_{54} & \psi_{55} & * & * & * & * \\
\psi_{61} & 0 & \psi_{63} & 0 & 0 & \psi_{66} & * & * & * \\
0 & \psi_{72} & 0 & \psi_{74} & 0 & 0 & \psi_{77} & * & * \\
\psi_{81} & \psi_{82} & 0 & 0 & 0 & 0 & 0 & \psi_{88} & * \\
\psi_{91} & 0 & \psi_{93} & 0 & \psi_{95} & 0 & 0 & 0 & \psi_{99}
\end{array}\right]<0
$$

$$
\begin{aligned}
& \psi_{11}=\left[\begin{array}{cc}
-W+Z_{1}+\varepsilon G_{1}^{T} G_{1} & * \\
-W+Z_{2}+\varepsilon G_{1}^{T} G_{1} & -Y+Z_{3}+\varepsilon G_{1}^{T} G_{1}
\end{array}\right], \psi_{22}=\left[\begin{array}{cc}
-Z_{1} & * \\
-Z_{2} & -Z_{3}
\end{array}\right], \psi_{31}=\left[\begin{array}{cc}
\varepsilon G_{2}^{T} G_{1} & \varepsilon G_{2}^{T} G_{1} \\
\varepsilon G_{3}^{T} G_{1} & \varepsilon G_{3}^{T} G_{1}
\end{array}\right], \\
& \psi_{33}=\left[\begin{array}{cc}
-\gamma^{2} I+\varepsilon G_{2}^{T} G_{2} & * \\
\varepsilon G_{3}^{T} G_{2} & -\gamma^{2} I+\varepsilon G_{3}^{T} G_{3}
\end{array}\right], \psi_{51}=\left[\begin{array}{cc}
W A \\
Y A+\delta_{0} S C+M+\delta_{2}(W-Y) & Y A+\delta_{0} S C
\end{array}\right], \\
& \psi_{52}=\left[\begin{array}{cc}
0 & 0 \\
\delta_{1} S C-\delta_{2} M & \delta_{1} S C
\end{array}\right], \psi_{53}=\left[\begin{array}{cc}
W B_{d} & W B_{f} \\
Y B_{d}+\delta_{0} S D_{d} & Y B_{f}+\delta_{0} S D_{f}
\end{array}\right], \psi_{54}=\left[\begin{array}{cc}
0 & 0 \\
\delta_{1} S D_{d} & \delta_{1} S D_{f}
\end{array}\right], \\
& \psi_{55}=\left[\begin{array}{cc}
-W & * \\
-W & -Y
\end{array}\right], \psi_{61}=\left[\begin{array}{cc}
0 & 0 \\
\alpha_{0} S C & \alpha_{0} S C
\end{array}\right], \psi_{63}=\left[\begin{array}{cc}
0 & 0 \\
\alpha_{0} S D_{d} & \alpha_{0} S D_{f}
\end{array}\right], \psi_{66}=\left[\begin{array}{cc}
-\alpha_{0} W & * \\
-\alpha_{0} W & -\alpha_{0} Y
\end{array}\right], \\
& \psi_{72}=\left[\begin{array}{cc}
0 & 0 \\
\alpha_{1} S C & \alpha_{1} S C
\end{array}\right], \psi_{74}=\left[\begin{array}{cc}
0 & 0 \\
\alpha_{1} S D_{d} & \alpha_{1} S D_{f}
\end{array}\right], \psi_{77}=\left[\begin{array}{cc}
-\alpha_{1} W & * \\
-\alpha_{1} W & -\alpha_{1} Y
\end{array}\right], \\
& \psi_{81}=\left[\begin{array}{cc}
0 & 0 \\
\alpha_{2}(W-Y) & 0
\end{array}\right], \psi_{82}=\left[\begin{array}{cc}
0 & 0 \\
-\alpha_{2} M & 0
\end{array}\right], \psi_{88}=\left[\begin{array}{cc}
-\alpha_{2} W & * \\
-\alpha_{2} W & -\alpha_{2} Y
\end{array}\right], \\
& \psi_{91}=\left[\begin{array}{cc}
N & 0 \\
0 & 0
\end{array}\right], \psi_{93}=\left[\begin{array}{cc}
0 & -I \\
0 & 0
\end{array}\right], \psi_{95}=\left[\begin{array}{cc}
0 & 0 \\
H^{T} W & H^{T} Y
\end{array}\right], \psi_{99}=\left[\begin{array}{cc}
-I & 0 \\
0 & -\varepsilon I
\end{array}\right] .
\end{aligned}
$$

Moreover, the desired filter parameters of (5) can be given by

$$
A_{F}=(W-Y)^{-1} M, B_{F}=(W-Y)^{-1} S, C_{F}=N
$$

Proof : Partitioning matrices $P$ and $U=P^{-1}$ into the following block forms:

$$
P=\left[\begin{array}{cc}
P_{1} & P_{2} \\
P_{2}^{T} & P_{3}
\end{array}\right], U=P^{-1}=\left[\begin{array}{cc}
U_{1} & U_{2} \\
U_{2}^{T} & U_{3}
\end{array}\right]
$$

where $P_{1} \in R^{n \times n}$ and $U_{1} \in R^{n \times n}$ are symmetric positive definite matrices, $P_{2}$ and $U_{2}$ are two non-singular matrices.

Define the invertible matrices as follows:

$$
J_{1}=\left[\begin{array}{cc}
U_{1} & I \\
U_{2}^{T} & 0
\end{array}\right], \quad J_{2}=\left[\begin{array}{cc}
I & P_{1} \\
0 & P_{2}^{T}
\end{array}\right]
$$

which implies that $P J_{1}=J_{2}$, and let $J_{1}^{T} Q J_{1}=\left[\begin{array}{cc}U_{1} Z_{1} U_{1} & U_{1} Z_{2}^{T} \\ Z_{2} U_{1} & Z_{3}\end{array}\right]$.

Performing congruence transformation to (10) by the transformational matrix $\Xi_{1}$, then pre- and post-multiplying the resulting LMI of the above transformation by the transformational matrix $\Xi_{2}$ and defining the following matrix variables: $U_{1}^{-1}=W, P_{1}=Y, P_{2} A_{F} U_{2}^{T}=M W^{-1}, C_{F} U_{2}^{T}=N W^{-1}, P_{2} B_{F}=S$, we can arrive at the result (11) in Corollary 1, where $\Xi_{1}=\operatorname{diag}\left\{\begin{array}{lllllll}J_{1}, & J_{1}, I, I, & J_{1}, & J_{1}, & J_{1}, & J_{1}, & I\end{array}\right\}$, $\Xi_{2}=\operatorname{diag}\left\{U_{1}^{-1}, \quad I, \quad U_{1}^{-1}, \quad I, \quad I, \quad I, \quad I, \quad I, \quad U_{1}^{-1}, I, \quad U_{1}^{-1}, I, \quad U_{1}^{-1}, I, \quad U_{1}^{-1}, I, \quad I, I\right\}$.

The transfer function matrix $T_{f}(z)$ of the RFDF as shown in (5):

$$
T_{f}(z)=C_{F}\left(z I-A_{F}\right)^{-1} B_{F}=N\left(z I-\left(P_{2} U_{2}^{T} U_{1}\right)^{-1} M\right)^{-1}\left(P_{2} U_{2}^{T} U_{1}\right)^{-1} S
$$


From $P^{*} P^{-1}=I$, it can be obtained that $P_{1} U_{1}+P_{2} U_{2}^{T}=I$ and $P_{2} U_{2}^{T} W=W-Y$. Moreover, the desired RFDF parameters can be given by (12).

This ends the proof.

Remark 2: Let $g=\gamma^{2}$. The sub-optimal robust filtering problem for discrete-time uncertain systems with one-step communication delay and measurement missing can be represented as follows:

$$
\min _{\mathrm{W}, Y, Z_{1}, Z_{2}, Z_{3}, M, N, S} g
$$

The parameters of the sub-optimal filters can be determined by (12), and the sub-optimal $H_{\infty}$ attenuation levels are given by $\gamma_{\text {opt }}=\sqrt{g^{*}}$, where $g^{*}$ are the sub-optimal solutions of the corresponding convex optimization problems.

\section{Simulation example}

In this section, a numerical example is proved to illustrate the effectiveness and applicability of the proposed method. Consider the system described by (1) with parameters as follows:

$$
\begin{gathered}
A=\left[\begin{array}{ccc}
0.5 & -0.6 & 0.7 \\
-0.2 & 0.4 & 0.5 \\
0.6 & 0.3 & -0.3
\end{array}\right], B_{d}=\left[\begin{array}{l}
0.2 \\
0.3 \\
0.5
\end{array}\right], B_{f}=\left[\begin{array}{c}
0.35 \\
0.45 \\
0.2
\end{array}\right], H=\left[\begin{array}{l}
0.1 \\
0.1 \\
0.1
\end{array}\right], \\
C=\left[\begin{array}{lll}
3.9 & 2.5 & 3.5
\end{array}\right], D_{d}=1.0, D_{f}=1.6, G_{1}=\left[\begin{array}{lll}
0.1 & 0 & 0.1
\end{array}\right], G_{2}=G_{3}=0.1, F(k)=0.5 .
\end{gathered}
$$

Let $\delta_{0}=0.2, \delta_{1}=0.7, \delta_{2}=0.1$. In other words, the measurements can be ideally transmitted over the network with probability 0.2 , one-step measurement delay can occur with probability 0.7 , and the measurements are missing with probability 0.1 . With the prescribed parameters, (11) can be solved by using the Matlab LMI toolbox. As a result, the minimum noise attenuation level bound is $\gamma_{\text {opt }}=1.0034$, and the parameters of RFDF are given by

$$
A_{F}=\left[\begin{array}{ccc}
0.1109 & -0.2836 & 0.3027 \\
-0.1205 & 0.0512 & 0.2058 \\
0.2459 & 0.1170 & -0.2768
\end{array}\right], B_{F}=\left[\begin{array}{c}
-0.0017 \\
0.0051 \\
0.0086
\end{array}\right], C_{F}=\left[\begin{array}{lll}
-0.0004 & 0.0001 & 0.0032
\end{array}\right] .
$$

For $k=0,1, . . ., 200$, the unknown input $d(k)$ is supposed to be random noise which is uniformly distributed over $[-0.5,0.5]$, and the fault signal $f(k)$ is given as:

$$
f(k)=\left\{\begin{array}{cc}
1, & k=60, \cdots, 140 \\
0, & \text { else }
\end{array}\right.
$$

By using (8), the threshold $J_{t h}=0.000423$ can be obtained. The simulation results indicate that the evaluation function $J(67)=0.00043884>J_{t h}$, so the appeared fault can be detected after 7 time steps. Fig.1 shows the generated residual signal $r(k)$ with stepwise fault signal. Fig.2 demonstrates the evolution of residual evaluation function, in which the blue dashed line stands for fault-free case and the red solid line for the fault case. The simulation results are given in Fig. 1 and Fig.2, from which effectiveness of the method is illustrated.

In addition, we can obtain $\frac{\left\|r_{e}(k)\right\|_{2}}{\|\psi(k)\|_{2}}=0.9028<\gamma=1.0034$. The simulation shows that the robustness of residual to unknown inputs and model uncertainties can be effective.

\section{Conclusions}

The problem of RFDF has been considered in this paper for a class of norm-bounded uncertain systems with communication delays and multiple measurement packet dropouts. The RFDF based on 
$H_{\infty}$ filtering has been used as residual generator. Then the design problem of RFDF has been reduced to $H_{\infty}$ filtering problem. A sufficient condition for the existence of the RFDF has been established by means of LMIs, and the parameters of the RFDF are determined directly by solutions of the LMIs. The simulation result has showed the effectiveness and applicability of the obtained approach.

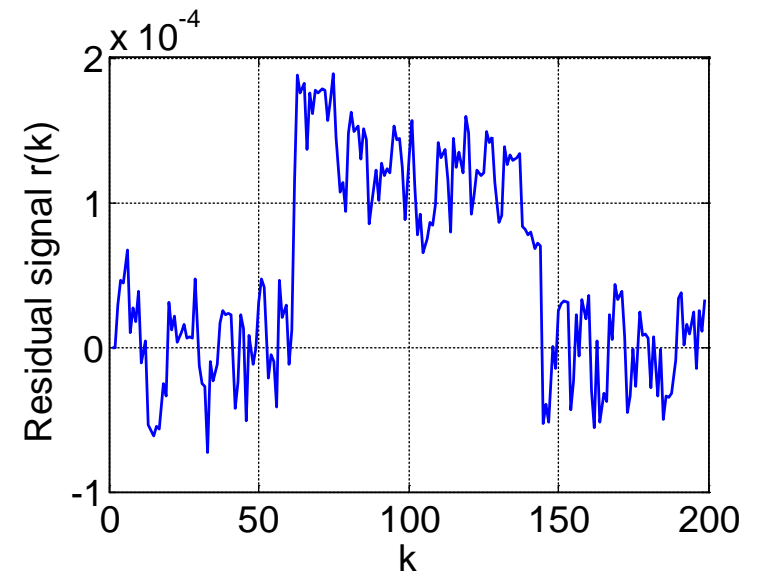

Fig.1 Residual $r(k)$ with Stepwise fault signal

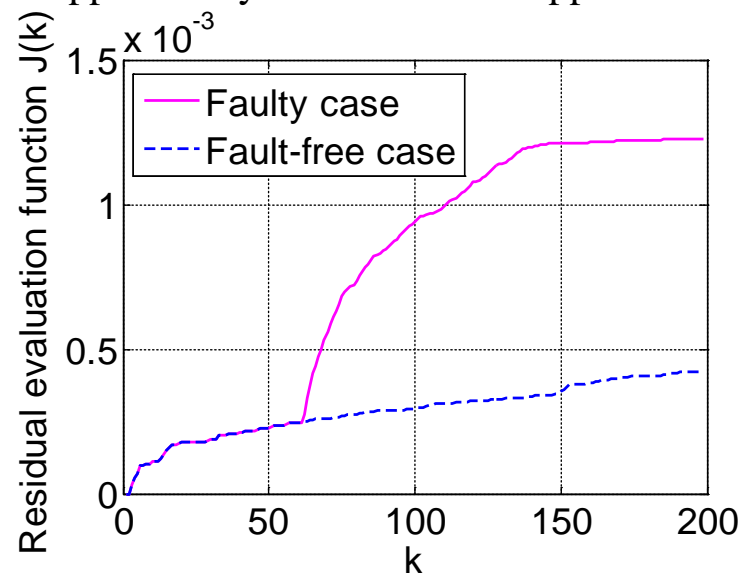

Fig.2 Evolution of residual evaluation $J(k)$

\section{References}

[1] Xu S X, Chen T W, Lam J. Robust $H_{\infty}$ filtering for uncertain Markovian jump systems with mode-dependent time delays [J]. IEEE Trans. Automatic Control, 2003, 48 (5):900-907.

[2] Wang Z D, Daniel W C, Liu X H. Robust filtering under randomly varying sensor delay with variance constraints [J]. IEEE Trans. On Circuits \& Systems, 2004, 51(6):320-326.

[3] Chen Li, Zhong MAI-Ying. Designing robust $H_{\infty}$ fault detection filter for singular time-delay systems with uncertainty [J]. Acta Automatica Sinica, 2008:34(8): 943-949.

[4] Zhong Maiying, Ding Steven X, Lam James, el at. An LMI approach to design robust fault detection filter for uncertain LTI systems [J].Automatic, 2003, 39(3):543-550.

[5] Wang Hongru, Wang Changhong, Gao Huijun. Robust fault detection for uncertain systems via LMIs [J]. Electric Machines and Control, 2005:9(5):461-565.

[6] Ruan Yu-bin, Yang Fu-wen, Wang Wu. Robust fault detection for networked systems with stochastic communication delays [J]. Journal of Fuzhou University (Natural Science Edition), 2008, 36(4):527-531.

[7] Wang Wu,Yang Fu-wen. $H_{\infty}$ filter design for discrete-time systems with missing measurements [J]. Acta Automatica Sinica, 2006, 32(1):107-111.

[8] Ruan Yu-bin, Wang Wu, Yang Fu-wen. Fault detection filter for networked systems with missing measurements [J]. Control Theory and Applications, 2009,26(3):291-295.

[9] He Xiao, Wang Zi dong, and Zhou Dong hua. Networked fault detection with random communication delays and packet losses. International journal of systems Science, 2008, 39(11):1045-1054.

[10] Wang Y Q, Ye H, Ding S X, Wang G Z, Zhou D H. Residual generation and evaluation of networked control systems subject to random packet droput.Automatica,2009,45(10):2427-2434.

[11] He X, Wang Z D, Zhou D H, Robust fault detection for networked systems with communication delay and data missing.Automatica,2009,45(11):2634-2639.

[12] Wang Zi-dong, Yang Fu-wen, Ho D C, et al. Robust $H_{\infty}$ filtering for stochastic time-delay systems with measurements [J].IEEE Transactions on signal processing, 2006, 54(7):2579-2587. 
[13] Yang Fu-wen, Wang Zi-dong, Hung Y S et al. $H_{\infty}$ Control for networked systems with random communication delays [J].IEEE Trans.on Automatic Control, 2006, 51(3):511-518. 